\title{
Reliable Radiation Technique to Minimize Ovarian Dose During Radiation Prophylaxis of Heterotopic Ossification
}

\author{
WALEED F. MOURAD ${ }^{1,2}$, SATYASEELAN PACKIANATHAN ${ }^{1}$, WEISI YAN ${ }^{3}$, \\ MAHBOUBEH PISHGOU ${ }^{2}$, JOHN K. MA ${ }^{1}$, SHERIF G. SHAABAN ${ }^{2}$, EDWARD M. MARCHAN ${ }^{2}$, \\ RUI HE ${ }^{1}$, PAUL N. MOBIT ${ }^{1}$, CHUNLI (CLAUS) YANG ${ }^{1}$ and SRINIVASAN VIJAYAKUMAR ${ }^{1}$ \\ ${ }^{I}$ Department of Radiation Oncology, University of Mississippi Medical Center, Jackson, MS, U.S.A.; \\ ${ }^{2}$ Department of Radiation Oncology, Erlanger Cancer Center - \\ University of Tennessee College of Medicine, Chattanooga, TN, U.S.A.; \\ ${ }^{3}$ Department of Radiation Oncology, University of South Alabama, Mobile, AL, U.S.A.
}

\begin{abstract}
Background/Aim: Scattered radiation during radiotherapy $(R T)$ directed at the hip joint poses concerns about ovarian function in patients of reproductive age. Here, we report the impact of using a split-beam technique (SBT) and different photon energies on the total ovary dose during radiation prophylaxis of heterotopic ossification (HO). Patients and Methods: This was a single-institution, retrospective study of 32-patients with traumatic acetabular fractures (TAF). All underwent surgery followed by CT-based$R T$ within $72 \mathrm{~h}$ in a single fraction of $700 \mathrm{cGy}$. Ipsilateral (IL) and contralateral $(C L)$ ovaries $(O V)$ were contoured separately and dose volume histograms (DVH) generated. Additional planning trials were created for each patient by utilizing a SBT medially and by using different photon energies (6-18 MV) to investigate the difference in ovary dose among these maneuvers. Results: The median Mean-dose delivered to ILOV was 59 cGy and the median Max-dose was 177 cGy. CLOV median Mean-dose was 6 cGy and median Max-dose was 10 cGy. SBT at the medial edge of the field led to a $27 \%$ and $22 \%$ dose reduction in the median Mean and Max. doses, respectively, to ILOV; $9 \%$ and $5 \%$ reduction was seen in the median Mean and Max. doses, respectively, to CLOV. Higher photon energies (10-18 MV) led to an additional $28 \%$ and $16 \%$ reduction in median Mean and
\end{abstract}

Presented at the American Society for Therapeutic Radiology and Oncology (ASTRO) 52th Annual Meeting 2014.

Correspondence to: Waleed F. Mourad, Department of Radiation Oncology, University of Mississippi Medical Center, Jackson, MS, U.S.A. Tel: +1 3477827886, e-mail: Waleed246@gmail.com

Key Words: Radiation therapy (RT), acetabular fracture, heterotopic ossification (HO), megavoltage photon, split-beam technique, ovarian dose, radiation prophylaxis, indomethacin.
Max. doses, respectively, to ILOV when compared to those from $6 \mathrm{MV}$. The CLOV median Mean dose was reduced by $18 \%$ and the Max. dose was reduced by $12 \%$. Conclusion: A biologically significant radiation dose is delivered to the ovaries during $\mathrm{HO}$ radiation prophylaxis at the hip joints. Ipsilateral ovarian dose could be reduced by half and contralateral by one-quarter by using CT-based treatment planning with a medial SBT and photon energies above $6 \mathrm{MV}$. We suggest using no more than $10 \mathrm{MV}$ to minimize neutron contamination. Those techniques should be the standard of care as it provides a reliable method for minimizing the radiation dose to the ovaries, consequently, maximizing female fertility preservation during $\mathrm{HO}$ radiation prophylaxis. All female patients in childbearing age should be fully informed about ovarian radiation exposure and possible temporary alteration in ova production and morphology.

Heterotopic ossification ( $\mathrm{HO}$ ) is the pathologic development of extra-skeletal bone in non-osseous tissues following exposure to severe burns, musculoskeletal trauma, and spinal cord injury (1). Posterior surgical approaches to the acetabulum, T-type acetabular fractures, a high injury severity score, a delay to surgery, a closed head injury, male gender, and previous history of heterotopic bone formation are among the risk factors that can contribute to the development of heterotopic ossification (HO) in injured tissues (2). The prophylactic use of indomethacin and/or single postoperative dose of external beam radiation therapy (RT) are two well established interventions to prevent development of HO (3). Prophylactic RT has been shown to be effective in reducing the risk of $\mathrm{HO}$ after open reduction and internal fixation (ORIF) of traumatic acetabular fractures (TAF) particularly in patients who were treated using a posterior surgical approach as it has been reported that $\mathrm{HO}$ development risk in such cases is up to $50 \%$ (4). Postoperative RT is most valuable and effective when 
administered within 3 days postoperatively owing to its ability to inhibit the proliferation of pluripotential mesenchymal cells that could potentially differentiate into osteoblastic stem cells $(5,6)$. Childs and Archdeacon et al., found no significant difference in terms of HO frequency or severity when comparing patients with acetabular fracture treated preoperatively or postoperatively with radiation therapy $(7,8)$. Popovic et al., Bird and Kang, reported that the incidence of HO after ORIF and RT or Indomethacin prophylaxis for TAF was $27 \%$ and $37 \%$, respectively and $12 \%$ after both RT combined with indomethacin. However, in control patients who received no prophylaxis, it was increased up to $58 \%(9,10)$. Using high energy photon beams and split-beam techniques (SBT) radiation to adjacent tissues can be minimized (11-15). Although the majority of patients at risk of $\mathrm{HO}$ are males, females are also susceptible to HO development. In their cases, the ovaries, being located within the pelvis, are always exposed to scatter radiation, which has the potential to affect their fertility. Being extremely sensitive to ionizing radiation, ovaries are highly susceptible to the damage from radiation induced apoptosis; Oocyte tolerance is estimated to be <2 Gy (16-24). Therefore, in this work we report techniques which can provide a treatment plan with optimal coverage of the targeted area while delivering the minimal ovarian dose.

\section{Patients and Methods}

Study objective. We conducted a retrospective study to evaluate the impact of different photon energies and dose delivery techniques in the total radiation dose received by ovaries after RT prophylaxis of HO. We hypothesized that by utilizing a SBT and by using higher photon energies (10 and $18 \mathrm{MV}$ ) we could reduce the scatter dose to the ovaries and consequently diminish the likelihood of any RTinduced ovarian damage.

Patient population. This study represents a single-institution; retrospective investigation performed at the University of Mississippi Medical Center, in Jackson, MS, U.S.A. and was fully approved by our Institutional Review Board.

Patient eligibility and design. Between 2008 and 2010, 32 female patients, all of whom had traumatic acetabular fractures (TAF) underwent surgery followed by RT.

Surgical treatment. All surgical procedures were performed by a fellowship trained, orthopedic trauma surgeon. All patients underwent open reduction and internal fixation (ORIF) via a posteriorly approached (Kocher-Langenbeck) surgical exposure with the patient in either lateral or prone position $(25,26)$. Early postoperative patient mobilization with toe-touch weight bearing as tolerated was initiated for all patients.

Radiation treatment. Postoperative RT was delivered within 72 hin a single fraction of $700 \mathrm{cGy}$, prescribed to the mid-plane, using MV photons. The fields included the soft tissues around the proximal femur and acetabulum without bone shielding. All patients underwent CT simulation and 2 portal images prior to their treatment (25-31). In all patients, the ipsilateral (IL) and contralateral (CL) ovaries (OV) were contoured separately as a region of interest and dose volume histograms (DVHs) generated. Additional planning trials were created for each patient by utilizing a SBT medially and by using different photon energies $(6,10$, and $18 \mathrm{MV}$ ) to investigate the effects of these maneuvers on the delivered dose to the ovaries (Figure 1A-C).

Medical treatment. Fifty percent (16/32 patients) of the whole cohort received RT with Indomethacin. Indomethacin was prescribed at the discretion of the surgeon at the dosage of $25 \mathrm{mg}$ three times per day after meals, beginning on postoperative Day 1 and continued for 6 weeks thereafter; however, patient compliance with daily intake was not verified nor were serum Indomethacin levels documented.

Follow-up. Patients had follow-up appointments at 2 weeks, 1 month, 3 months, 6 months, and 1 year from the day of discharge. Standard hip X-rays (AP, PA, and oblique) were obtained for evaluation of HO per the Brooker classification (32), avascular necrosis of the femoral head, or loosening, malunion, or non-union of the fracture. Computed tomography of the pelvis was obtained where considered clinically appropriate. All patient medical records and X-rays were reviewed to determine the efficacy of the RT in preventing HO. The presence or absence of $\mathrm{HO}$ was verified by three independent reviewers: a radiologist, an orthopedic surgeon, and a radiation oncologist.

Hypothesis and study endpoints. In this study, we hypothesized that by utilizing SBT and by using higher photon energies (10 and 18 MV) we could reduce the scatter dose to the ovaries and therefore diminish any RT-induced ovarian damage.

Statistical methods. Univariate and multivariate regression analyses were used to compare ovarian doses received in the different groups, after adjusting for all other factors that could potentially affect $\mathrm{HO}$ formation (body mass index (BMI), separation, type of fracture, and surgical approach to fracture exposure).

\section{Results}

Thirty-two female patients with a mean age of 41 years (Table I summarizes the patients' characteristics) were evaluated. Motor vehicle crashes (MVC) (100\%) was the cause of the fractures in all our patients. The median BMI was 27 (range $=19-45$ BMI). Posterior acetabular and combined posterior wall and T-shaped fractures were the most common types of injury seen in the majority of patients. All patients underwent ORIF via the KocherLangenbeck approach; thereafter, all underwent CT-based simulation and 3-D RT planning.

At a median follow-up of 2 years for the whole cohort, the incidence of $\mathrm{HO}$ in all patients was only $6.25 \%(2 / 32)$ with RT with/without indomethacin. The mean field separation for RT was $23 \mathrm{~cm}$. The irradiated tissue volume mean for all patients was $4,248 \mathrm{ml}$. All patients were treated at $100 \mathrm{~cm}$ $\mathrm{SAD}$, with a mean open field area on the skin of $153 \mathrm{~cm}^{2}$. 
Table I. Patients' characteristics.

\begin{tabular}{lc}
\hline Patients & $\mathrm{N}=32$ \\
\hline MVC & $100 \%$ \\
Female & $100 \%$ \\
Age & $32(18-56)$ \\
BMI & $27(19-45)$ \\
Left hip vs. right hip & $40 \%$ vs. $60 \%$ \\
Separation & $23(16-36)$ \\
Mean (Mean dose) cGy Ipsi. vs. contralateral ovary & $59 v s .6$ \\
Mean (Maximum dose) cGy Ipsi. $v s$. contralateral ovary & $177 v s .10$ \\
Fracture to Surgery in days & $2(0-14)$ \\
Fracture to RT in days & $4(2-16)$ \\
Surgery to RT in days & $2(1-3)$ \\
Indomethacin & $50 \%(16 / 32)$ \\
CT-based 3-DRT & $100 \%$ \\
700 cGy & $100 \%$ \\
Posterior wall fracture & $50 \%(16 / 32)$ \\
Posterior wall and T-shaped fracture & $25 \%(8 / 32)$ \\
T-shaped fracture & $25 \%(8 / 32)$ \\
Kocher-Langenbeck approach & $100 \%$ \\
HO & $6.25 \%(2 / 32)$ \\
Brooker Grade I\&II & $100 \%$ \\
\hline
\end{tabular}

MVC: Motor vehicle crash; CT: computerized tomography; cGy: centiGray; 3-DRT: three-dimensional radiation therapy; BMI: body mass index; HO: heterotopic ossification.

In reviewing the DVHs for all patients, it was noted that the mean (Mean dose) delivered to the ipsilateral ovaries was 59 cGy while the mean (Max. dose) was 177 cGy. For the contralateral ovaries, the mean (Mean dose) was $6 \mathrm{cGy}$ and the mean (Max. dose) was $10 \mathrm{cGy}$. The radiation doses to the ovaries from the CT simulation \& 2 portal images were computed at $4 \& 1.5 \mathrm{cGy}$, respectively.

After applying a beam split at the medial edge of the field, there was $27 \%$ and $22 \%$ dose reduction in the mean Mean and Max. doses, respectively, delivered to the ipsilateral ovaries and a 9\% and 5\% reduction in the mean Mean and Max. doses, respectively, to the contralateral ovaries.

By using higher photon energies (10 and $18 \mathrm{MV}$ ) rather than standard $6 \mathrm{MV}$ photons, an additional 19-36\% reduction in the ipsilateral ovaries' Mean dose and $11-22 \%$ dose reduction in the Max. dose were achieved. In the contralateral ovaries, similar proportional reductions were noted by using higher beam energies, $16-41 \%$ dose reduction in the Mean dose and 19-43\% reduction in the Max. dose. In all cases higher energies provided greater reduction.

The univariate regression test was applied first to test for an association between ovarian dose reduction and SBT and different photon energies before adjusting for other factors. There was a significant reduction in the ovarian dose $(p<0.001)$ with SBT. Further, multivariate regression analysis confirmed the significant reduction in ovarian dose after adjusting for all other factors (age, BMI, separation, size of the RT field, types of fracture and surgical exposures) that could potentially impact the ovarian dose. In reviewing the DVHs for all patients, it was noted that the median (Mean dose) delivered to the IL OV was $59 \mathrm{cGy}$ (range=6262 cGy) and the median (Max dose) was 177 cGy (range $=7-650 \mathrm{cGy}$ ). Similarly, the CL OV median (Mean dose) was $6 \mathrm{cGy}$ (range=1-11 cGy) and the median (Max dose) was $10 \mathrm{cGy}$ (range $=1-21 \mathrm{cGy}$ ). When a beam split was applied at the medial edge of the field, there was a $27 \%$ and $22 \%$ dose reduction in the mean and max. dose, respectively, delivered to the IL OV and a $9 \%$ and $5 \%$ reduction in the mean and max. dose, respectively, to the CL OV. Furthermore, when higher energies (10-18 MV) were compared to $6 \mathrm{MV}$, an additional $28 \%$ and $16 \%$ reduction in mean and max. doses, respectively, of the IL OV were achieved. Moreover, the CL OV mean dose was reduced by $18 \%$ and the maximum dose was reduced by $12 \%$ (Table II and Figure 2A and B).

\section{Discussion}

$\mathrm{HO}$ is the pathological process of bone formation in nonosseous tissues following trauma and/or surgical intervention. This misplaced growth occurs between muscle planes and not actually within the muscle fibers themselves (32). HO was first identified and reported in the literature in 1883 by Riedel (33), a German physician. It was later observed by the French physicians Dejerne and Ceillier (34) in patients suffering traumatic paraplegia in World War I. There are several factors that appear critical to HO formation - a traumatic injury, cellular signals from the site of injury, a supply of mesenchymal cells, and the appropriate tissue microenvironment - all appear to be factors that may contribute to the development of $\mathrm{HO}$ in injured tissues (1). The most common procedures that result in HO are: ORIF of an acetabular fracture or a total hip arthroplasty (THA) (9). Many clinicians recommend prophylactic RT and/or non-steroidal anti-inflammatory medications (NSAIDs) to prevent HO. Radiation therapy presumably decreases the risk of $\mathrm{HO}$ by inhibiting the proliferation of pluripotential mesenchymal cells that could potentially differentiate into osteoblastic stem cells and is usually given postoperatively, within $72 \mathrm{~h}$ of surgery or preoperatively (3-10). We hypothesized that by utilizing a SBT medially and/or by using higher photon energies (10 and $18 \mathrm{MV}$ ), we could minimize the scatter dose to the ovaries and, consequently, diminish any RT-induced short and long term ovarian damage. In order to achieve minimal medial dose divergence at the beam edge, a split beam technique (SBT), sometimes called a half-beam block (HBB), is often used. In this method, the beam is split along the plane containing the central axis inhibiting any geometric divergence of the 

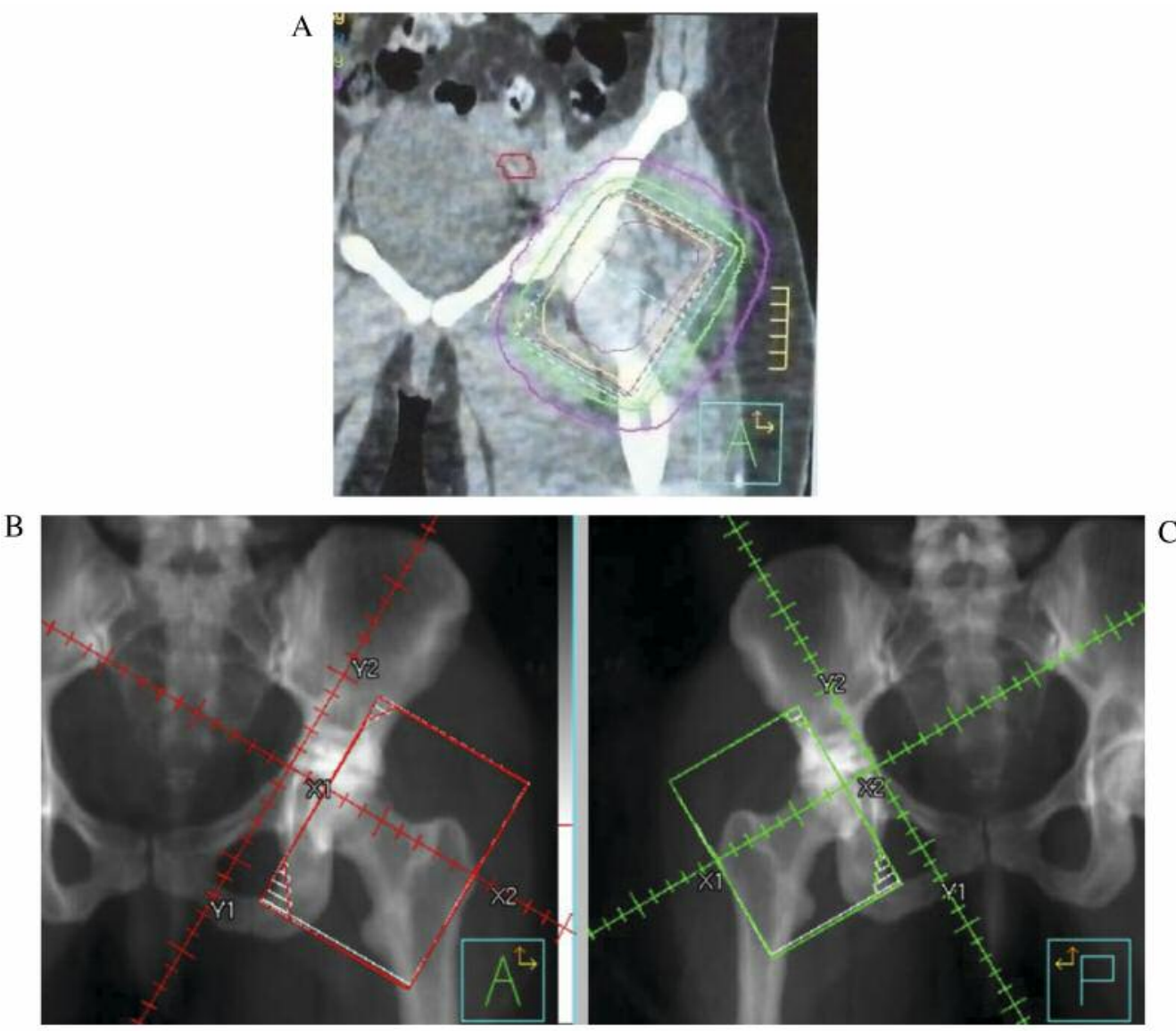

Figure 1. A, B, C. CT based planning and split-beam technique [(SBT), e.g. half-beam block], where the isocenter was placed at the acetabulum to minimize beam divergence into the pelvis.

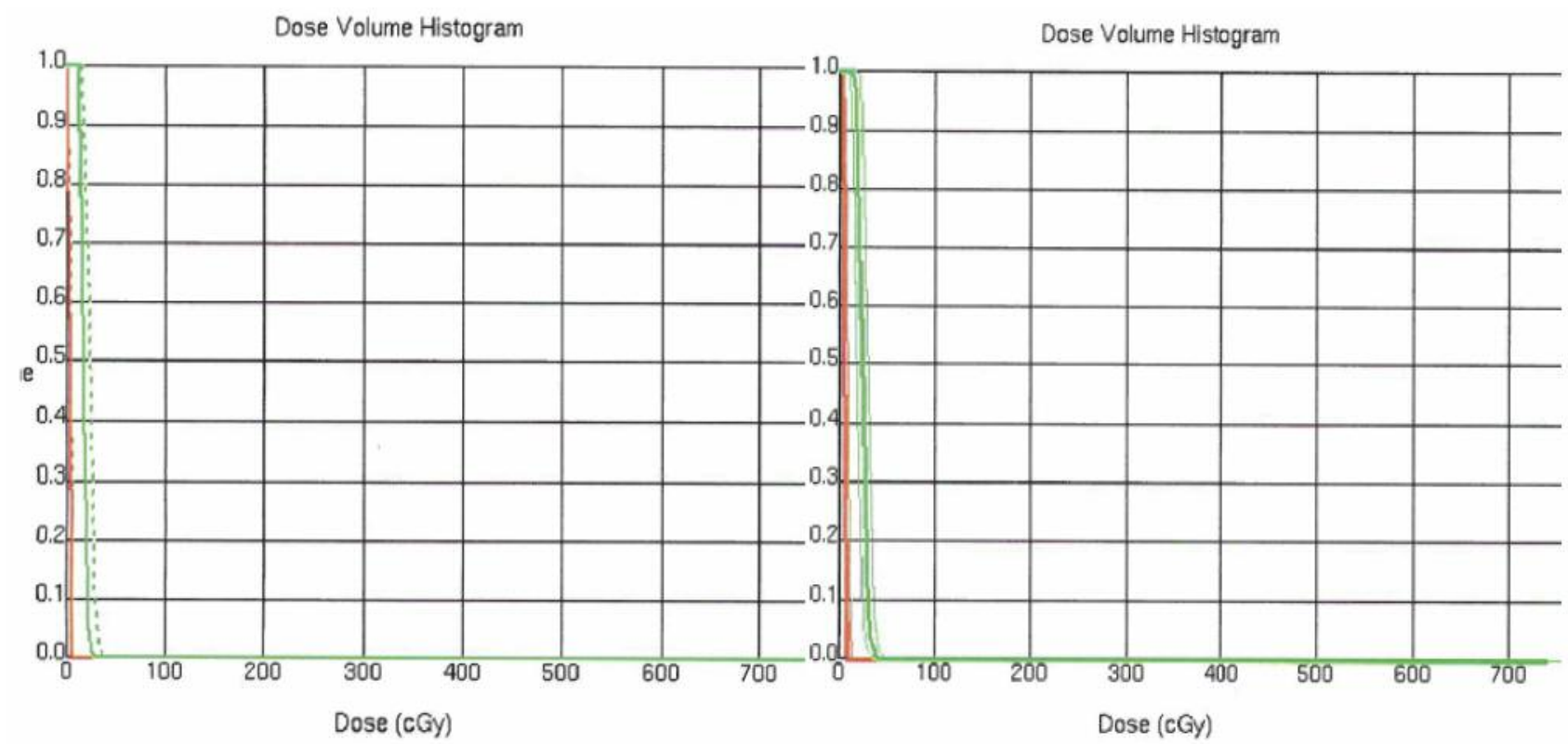

Figure 2. A, B. DVH reflecting the computed dose reduction (red line) on CLO and ILO by applying SBT and 10× MV. 
Table II. Percentage of dose reduction with beam-split technique (SBT) and different photon energies (10 and 18 MV) on ovarian dose.

\begin{tabular}{lcccc}
\hline Delivered Mean dose (cGy) & Mean dose IL.OV (59) & Max. dose IL. OV (177) & Mean dose CL.OV (6) & Max. dose CL. OV (10) \\
\hline SBT & $27 \%$ & $22 \%$ & $9 \%$ & $5 \%$ \\
lOX & $28 \%$ & $16 \%$ & $18 \%$ & $12 \%$ \\
18 X & $33 \%$ & $20 \%$ & $20 \%$ & $15 \%$ \\
Total dose reduction & $55 \%$ & $38 \%$ & $27 \%$ & $17 \%$ \\
\hline
\end{tabular}

Dose reduction-SBT $(p<0.001)$; Dose reduction - Energy $(p<0.001)$.

beams at the split line (11). This technique is often employed in matching one radiation field to another. By applying a $\mathrm{HBB}$ in the medial edge of the pelvic AP/PA fields in $\mathrm{HO}$ prophylaxis, much of the dose that can potentially diverge into the pelvis is diverted laterally.

Radiotherapy for all pre-menopausal female patients should be delivered carefully so as to minimize the ovarian dose as it can cause gonadal failure and impact female fertility. Direct total body, low abdominal, pelvic, or spinal radiation, and scatter radiation from fields distant to the ovaries all can impact the ovaries (19, 20). The radiosensitivity and vulnerability of the oocyte to radiation induced cell death varies according to its growth phase, age of the patient and number of RT fractions (20). The granulosa cells (GCs) which line and support the growing follicles, seem to be the initial target for radiation injury. Within a few hours of irradiation, before any detectable changes are even seen in the oocyte, pyknosis - indicating cell death - can be seen in GCs. Consequently, there is loss of oocyte viability with atrophy of the follicle (21).

Acute ovarian failure (AOF) can be defined as complete or nearly complete disappearance of primordial follicles following receipt of a dose of irradiation or chemotherapy sufficient to cause permanent sterility during or shortly after treatment, whereas premature ovarian failure (POF) represents a transient infertility status, after receiving low radiation doses, in older women who have more radioresistant, less dividing follicles. Nonetheless, it can shorten the reproductive period with subsequent loss of ovarian function before the age of 40 years (22). It is postulated that a dose of less than $2 \mathrm{~Gy}$ is sufficient to inactivate half of the oocyte population, while a dose of more than $6 \mathrm{~Gy}$ will produce irreversible ovarian failure (23). Owing to the low primordial follicle pool and the high incidence of radiation-induced chromosome damage in the oocytes, older patients are at higher risk, compared with younger women, for potential development of infertility after radiotherapy (24). Therefore, the risk of premature ovarian insufficiency and subsequent infertility is directly proportional to the cumulative dose of pelvic radiotherapy delivered. Those of younger age are less likely to be affected due to the larger oocyte pool. In addition, radiotherapy fractionation should be taken into consideration when determining radiation effect on ovaries, as a single dose will be more toxic compared to fractionated doses (16-24). Wallace et al., described the first model to predict the age of premature ovarian failure after treatment with a known dose of radiotherapy and reported that the effective sterilizing dose at birth, 10 years, 20 years and 30 years is $20.3 \mathrm{~Gy}$, 18.4 Gy, 16.5 Gy, and 14.3 Gy, respectively $(19,20)$.

Radiation to oocytes could also lead to hereditary effects. The International Commission on Radiological Protection (ICRP) estimates the probability per caput for severe radiation induced hereditary effects to be $0.6 \times 10^{-2}$ per Gy. Thus, a beam split with high energy during treatment of $\mathrm{HO}$ would lead to a reduction from a maximum risk of 35 per 10,000 children to 16 per 10,000 children. Different methods of fertility preservation have been reported, including chemoprotection, ovariopexy, cryopreservation of ovarian tissue, and induced maturation of ovarian follicles. However, those modalities are not always available in the available time prior to initiation of radiation therapy (16-24). Accurate localization of ovaries location is very critical during planning to determine the ovarian dose delivery during radiation and, on the other hand, to detect any significant variation in position might occur. The latter was confirmed by Nicholson et al., who evaluated the ovarian location of 12 women of childbearing age who underwent pelvic magnetic resonance imaging (MRI) with full and empty bladders (17).

In summary, we hypothesized that by employing (SBT) medially and/or by using higher photon energies (10 and $18 \mathrm{MV}$ ), we could minimize the scatter dose to the ovaries, and therefore diminish RT-induced ovarian damage. We were able to minimize the ovarian dose by applying SBT and higher photon energies. There was a significant reduction (25-55\%) of ovarian dose by utilizing these available facilities. Utilization of SBT and $\geq 10 \mathrm{MV}$ photon beams led to a smaller scatter RT doses compared to lower energy $6 \mathrm{MV}$ photons, probably owing to the higher energy beams' ability to "forward scatter" rather than "side scatter". HBB techniques can also be employed to achieve minimal dose divergence at the beam 
edge; applying a HBB in the medial edge of the pelvic AP/PA fields in $\mathrm{HO}$ prophylaxis appears to reduce much of the dose which can diverge into the ovaries, bowel, and bladder (11). Use of CT simulation, 3-D treatment planning, SBT, and photon energies greater than $6 \mathrm{MV}$ has a significant reduction in ovarian RT scatter dose via minimizing the penumbra and lateral scatter dose from lower energy beams (11).

\section{Conclusion}

A biologically significant radiation dose is delivered to the ovaries during HO prophylaxis at the hip joints. This dose could be reduced to the IL OV by half and the CL OV by one-quarter by using 3 D-CT simulation and planning, followed by medial beam split technique and photon energies above $6 \mathrm{MV}$. We suggest using no more than $10 \mathrm{MV}$ to minimize neutron contamination. We urge that these techniques be the standard of care as they provide a reliable method for minimizing the radiation dose to the ovaries. All female patients especially those of childbearing age should be fully informed of the consequences of ovarian scatter radiation exposure and possible temporary alteration in ova production and morphology.

\section{Conflicts of Interest}

All Authors do not have any conflicts of interest.

\section{References}

1 Kaplan FS, Glaser DL, Hebela N and Shore EM: Heterotopic ossification. J Am Acad Orthop Surg 12: 116-125, 2004.

2 Shehab D, Elgazzar AH and Collier BD: Heterotopic ossification. J Nucl Med 43: 346-353, 2002.

3 Burd TA, Lowry KJ and Anglen JO: Indomethacin compared with localized irradiation for the prevention of heterotopic ossification following surgical treatment of acetabular fractures. J Bone Joint Surg Am 83: 1783-1788, 2001.

4 Haas ML, Kennedy AS, Copeland CC, Ames JW, Scarboro M and Slawson RG: Utility of radiation in the prevention of heterotopic ossification following repair of traumatic acetabular fracture. Int J Radiat Oncol Biol Phys 45: 461-466, 1999.

5 Pakos EE, Pitouli EJ, Tsekeris PG, Papathanasopoulou V, Stafilas $\mathrm{K}$ and Xenakis TH: Prevention of heterotopic ossification in highrisk patients with total hip arthroplasty: the experience of a combined therapeutic protocol. Int Orthop 30: 79-83, 2006.

6 Balboni TA, Gobezie $\mathrm{R}$ and Mamon HJ: Heterotopic ossification: Pathophysiology, clinical features, and the role of radiotherapy for prophylaxis. Int J Radiat Oncol Biol Phys 65: 1289-1299, 2006.

7 Childs HA 3rd, Cole T, Falkenberg E, Smith JT, Alonso JE, Stannard JP, Spencer SA, Fiveash J, Raben D, Bonner JA, Westfall AO and Kim RY: A prospective evaluation of the timing of postoperative radiotherapy for preventing heterotopic ossification following traumatic acetabular fractures. Int J Radiat Oncol Biol Phys 47: 1347-1352, 2000.
8 Archdeacon MT, Heurle DA, Nicole N and Budde B: Is Preoperative Radiation Therapy as Effective as Postoperative Radiation Therapy for Heterotopic Ossification Prevention in Acetabular Fractures? Clin Orthop Relat Res 472: 3389-3394, 2014.

9 Baird EO and Kang QK: Prophylaxis of heterotopic ossification. J Orthopaed Surg Res 4: 12, 2009.

10 Popovic M, Agarwal A, Zhang L, Yip C, Kreder HJ, Nousiainen MT, Jenkinson R, Tsao M, Lam H, Milakovic M, Wong E and Chow E: Radiotherapy for the prophylaxis of heterotopic ossification: A systematic review and meta-analysis of published data. Radiother Oncol 113: 10-17, 2014.

11 Khan FM: Physics of Radiation Therapy (4th ed). Philadelphia, PA, Lippincott Williams \& Wilkins, 2009.

12 Mourad WF, Ma JK, Yang CC, He R, Ryniak M, Shourbaji RA, Mobit PN, Santoro J, Packianathan S, Jennelle R and Vijayakumar S: Higher energy megavoltage photon and split beam technique minimize the testicular dose during radiation prophylaxis of heterotopic ossification. Int J Radiat Oncol Biol Phys 90: s684, 2014.

13 Mourad WF, Shourbaji RA and Packianathan S: Testicular dose during radiation prophylaxis of heterotopic ossification. American Radium Society annual meeting 2011.

14 Mourad WF, Shourbaji RA, Baird MC, Dieck G, Edwards J, Mobit P, Claus CC, Hu KS and Harrison LB: Ovarian radiation dose during heterotopic ossification radiation prophylaxis. Int $\mathrm{J}$ of Radiat Oncol Biol Phys 81: s640, 2011.

15 Mourad WF, Ma JK, Packianathan S, Yan W, Shaaban SG, Marchan EM, Abdallah LE, He R, Mobit PN, Yang CC and Vijayakumar S: Testicular Dose during Prophylaxis of Heterotopic Ossification with Radiation Therapy. In Vivo 31: 461-466, 2017.

16 Tease $\mathrm{C}$ and Fisher G: The influence of maternal age on radiation induced chromosome aberrations in mouse oocytes. Mutat Res 262: 57-62, 1991.

17 Nicholson R, Coucher J, Thornton A and Connor F: Effect of a full and empty bladder on radiation dose to the uterus, ovaries and bladder from lumbar spine $\mathrm{CT}$ and X-ray examinations. $\mathrm{Br}$ J Radiol 73: 1290-1296, 2000.

18 Sonmezer M and Oktay K: Fertility preservation in female patients. Hum Reprod Update 10: 251-266, 2004.

19 Wallace WH, Anderson RA and Irvine DS: Fertility preservation for young patients with cancer: Who is at risk and what can be offered? Lancet Oncol 6: 209-218, 2005.

20 Wallace WH, Thomson AB, Saran F and Kelsey TW: Predicting age of ovarian failure after radiation to a field that includes the ovaries. Int J Radiat Oncol Biol Phys 62: 738-744, 2005.

21 Lee SJ, Schover LR, Partridge AH, Patrizio P, Wallace WH, Hagerty K, Beck LN, Brennan LV and Oktay K: American Society of Clinical Oncology recommendations on fertility preservation in cancer patients. J Clin Oncol 24: 2917-2931, 2006.

22 Sklar CA, Mertens AC, Mitby P, Whitton J, Stovall M, Kasper C, Mulder J, Green D, Nicholson HS, Yasui Y and Robison LL: Premature menopause in survivors of childhood cancer: A report from the childhood cancer survivor study. J Natl Cancer Inst 98 : 890-896, 2006.

23 Stroud J, Mutch D, Rader J, Powell M, Thaker PH and Grigsby PW: Effects of cancer treatment on ovarian function. Fertil Steril 92: 417-427, 2009. 
24 Jensen JR and Morbeck DE: Fertility Preservation. Mayo Clin Proc 86: 45-49, 2011.

25 Mourad WF, Packianathan S, Shourbaji RA, Russell G, Zhang Z, Khan MA, Graves M, Jennelle R and Vijayakumar S: The Impact of BMI on Heterotopic ossification. Int $\mathrm{J}$ Radiation Oncol Biol Phys 82: 831-836, 2012.

26 Mourad WF, Packianathan S, Shourbaji RA, Russell G, Zhang Z, Khan MA, Graves M, Jennelle R and Vijayakumar S: The Impact of morbid (Class III) obesity on heterotopic ossification. Pract Radiat Oncol 2: 1-6, 2012.

27 Mourad WF, Packianathan S, Shourbaji RA, Russell G, Zhang Z, Khan MA, Graves M, Jennelle R and Vijayakumar S: A prolonged time interval between trauma and prophylactic radiation therapy significantly increases the risk of heterotopic ossification. Int J Radiation Oncol Biol Phys 82: 339-344, 2012.

28 Mourad WF, Saad AA, Packianathan S, Baird MC, Shourbaji RA, Russell G, Zhang Z, Caudell JJ, Jennelle R, Vijayakumar S and Hussein MA: Post-radiation therapy sarcoma. J Clin Oncol 28: e20523, 2010.

29 Mourad WF, Packianathan S, Baird MC, Shourbaji RA, Russell G, Khan MA, Akhtar I, Cheng S, Caudell JJ, Jennelle R and Vijayakumar S: Radiation-induced malignancy after prophylactic radiation therapy in prevention of heterotopic ossification. Int J Radiat Oncol Biol Phys 78: sP610, 2010.

30 Mourad WF, Packianathan S, Shourbaji RA, Russell G, Khan MA and Vijayakumar S: Radiation-induced sarcoma following radiation prophylaxis of heterotopic ossification. Pract Radiat Oncol 2: 151-154, 2012.
31 Mourad WF, Packianathan S, Ma JK, Yang CC, Shourbaji RA, He R, Zhang Z, Kanakamedala MR, Khan MA, Mobit P, Katsoulakis E, Nabhani T, Jennelle R, Russell GV and Vijayakumar S: Computerized tomography-based radiotherapy improves heterotopic ossification outcomes. Bone 57: 132-136, 2013.

32 Brooker AF, Bowerman JW, Robinson RA and Riley Jr LH: Ectopic ossification following total hip replacement: incidence and a method of classification. JBJS 55: 1629-1632, 1973.

33 Riedel B: Demonstration line durch ach Hagiges Umhergehen total destruirten kniegelenkes von einem patienten mitstichverletzing des ruckans. Verh Dtsch Gesellschaft Chirurg 12: 93, 1883.

34 Dejerine A and Ceillier A: Para-osteo-arthropathies des paraplegigues par lesionmedullarie; etude clinique et radiographique. Ann Med, 1918.
Received September 12, 2017

Revised October 12, 2017

Accepted October 17, 2017 